on the following topics: bulk single crystal growth of device graded substrate materials; bulk material characterization; processing and device properties; growth and characterization of heterostructures; material for optical transmitters; materials for optoelectronic integration; and new materials and layered structures for contacts, interconnects, insulation and passivation.

The following papers were a mong those presented by 28 invited speakers:

"Advanced LEC GaAs Crystal Growth for Optoelectronic ICs" by T. Fukuda (Japan)

"Defect Characterization by Magnetic Resonance and Infrared Spectroscopy" by J. Schneider (West Germany)

"Electron Nuclear Double ResonanceOptically Detected Electron Nuclear Double Resonance" by J.M. Spaeth (West Germany)

"Transport Properties in Modulation Doped Heterostructures and Quantum Wells" by G. Weimann (West Germany) "Epitaxial'Growth of II-VISemiconductors for Telecommunications" by R.D. Feldman (United States)

"Characterization of Heterostructures and Multilayer Stacks by Optical Methods: Kinetic and Spectroscopy Ellipsometry, Microphotoluminescence, X-Ray Reflectrometry" by J.B. Theeten (France)

"Material and Technology Requirements for GaAs Optoelectronic Integration" by F. Brillouet (France)

"Approach to Improved Materials Structures for Contacts to Compound Semiconductor Devices" by L.F. Eastman (United States)

"Telecommunication Systems" by J. Jerphagnon (France)

Special emphasis was given to the topic "Correlation of Bulk Material - Processing - Device Properties," which was discussed in a special rump session. It was agreed that this important issue should be suggested as a topic for a future E-MRS symposium.

\section{E-MRS Symposium Covers Magnetic Thin Films}

Symposium E at the E-MRS Spring Meeting held in Strasbourg, France during the week of June 16, 1986 had as its topic "Magnetic Thin Films." The symposium organizers, led by R. Krishnan (CNRS, France), included P.G. Grundy (Salford University, UK), H. Hoffmann (Universität Regensburg. West Germany), and J.C. Lodder (Twente University of Technology, Netherlands).

The two-day scientific program, attended by over 50 scientists from industry and national laboratories, included sessions on soft films, multilayers, and applications, and a panel discussion on monolayer and multilayer films. Each session began with an invited paper which set the pace for subsequent contributed papers.
Prof. Hoffmann's invited talk on soft magnetic film properties started with a brief survey of the work dore in the 1960 s. He dealt with ripples in permalloy and finally discussed the implications in amorphous soft films. The next invited paper by Wettling et al., a stimulating one on surface spin wave studies in magnetic multilayers, demonstrated the use of Brillouin light scattering to investigate the collective excitations in multilayers, a subject of intense interest today. The role of anisotropic stack was also theoretically investigated. Experimentors can now obtain such samples. The last invited talk, representing industry and given by Bernstein et al., was on perpendicular recording properties of amorphous $\mathrm{Tb}$-Fe and Gd-Fe films. They described the correlation between the recording properties and the magnetization process which they studied using magneto-optical techniques.

The following is a brief topical account of contributed papers:

Thin films. Five papers dealt with the domain structure, exchange coupling between a magnetically hard and a soft film, and an ion beam mixing technique to produce amorphous films.

Multilnyers. Eight papers considered various aspects such as structure of sandwich-type films, surface magnetism, investigations using FMR and NMR and spin-resolved photoemission studies. The work described both epitaxial layers and polycrystalline ones dealing with the effect of layer thickness on properties. They brought out the importance of in silu characterizations. Applications. Eight papers considered topics ranging from amorphous metallic films to ferrimagnetic spinel and garnet-type films. Stability in amorphous rare earth-transition metal films was discussed. A new assisted-CVD technique to prepare spinel films for recording applications was described. Magneto-optical properties both in metallic multilayers and garnet films were presented. Ion implantation problems in YIG and barium ferrite were discussed. A final paper dealt with Bloch line containing bubble wall states.

The panel discussion on monolayers and multilayers was an interesting, exciting session. Several aspects were discussed, including: various techniques of preparation and their merits and demerits, a wide spectrum of characterization techniques for obtaining a better understanding of the interface, coupling, and so on.

The participants seemed satisfied with the program, but according to symposium organizer Krishnan, much more could be accomplished, given more time. There are many conferences on magnetism and magnetic materials, and many could question the need for the E-MRS June meeting. Says Krishnan, "Yes, there are many conferences each year in many parts of the world, but they tend to be either too large or too expensive for many. The result is that often the same people attend, and therefore a real exchange of ideas between those active in the field is not really achieved. Under these circumstances, I believe that a medium-sized forum in Europe is indeed most welcome, even if it is an annualevent. It is necessary that European researchers meet periodically to confront and compare their ideas in order to effectively plan their research."

\section{E-MRS Plans 1987 Meeting}

E-MRS has begun to solicit papers for its 1987 meeting to be held in June 1987. Papers are being solicited for the symposia described below. The deadline for submitting abstracts is March 15, 1987. For more information, contact the symposium chairs listed at the end of each symposium description.

\section{Amorphous Hydrogenated Carbon Films}

This three-day symposium will consist of contributed and invited papers on the preparation, structure, properties, and applications of hard amorphous hydrogenated carbon (a-C:H) films. This metastable carbonaceous material is finding increasing application as an optical, dielectric, tribological, or corrosion-resistant coating and as a material for wall conditioning in fusion devices. Joint plenary talks will be organized with the "Symposium on Surface Engineering." Papers are solicited in the following areas:

\section{Preparation}

Process characterization (plasma chemistry, surface reactions)

Bonding and structure

Properties (a-C:H bulk and film/substrate interface)

Post deposition modification (heat treatment, ion beams)

Applications

Related materials (a-C, a-C:F, C/group IValloys, CVD-diamond films)

\section{Symposium Chairs:}

P. Koidl

Fraunhofer-Institut für

Angewandte Festkörperphysik

Eckerstrasse 4

D-7800 Freiburg

Federal Republic of Germany

(0761)2714280

\section{P. Oelhafen}

Institut für Physik

Universität Basel

Klingelbergstrașse 82

$\mathrm{CH}-4056$ Basel

Switzerland

(061)442040 
Corrrelation Between Material Properties, Processing, and Device Performances in Compound Semiconductors

This symposium will discuss material properties of compound semiconductors that are directly linked to processing technologies and device performances. The major compound semiconductor devices will be addressed separately for their specific material requirements. Uniformity and reproducibility over a large area for monolithic integration on single crystal substrates as well as thin film structures will be discussed. State-of-the-art silicon compatible structures as a potential competitor for compound semiconductor devices will also be featured. Papers are solicited on the following topics:

- Growth and characterization of device quality substrates and thin film structures (for FET, TEGFET, lasers)

- Specific material properties needed for: Integrated circuits (uniformity, reproducibility, substrate size limitations)

Optoelectronic integration

- Competing silicon compatible material:

Compound semiconductor thin film growth on Si substrate

$\mathrm{Si}$-SiGe heterostructures

Symposium Chairs:

P.A. Glasow

Siemens A.G.
Central Research and Development

Research Laboratories Munich-Erlangen

Paul. Gossen strasse 100, D8520

Erlangen

Federal Republic of Germany

(9131)731225

Y.I. Nissim

CNET

Laboratoire de Bagneux

196 , rue H. Ravera

92220 Bagneux

France

33.1.45295319

Photon, Beam, and Plasma Based

Chemical Processing

This three-day symposium will highlight emerging technologies using lasers, incoherent light, charged particle beams, and plasmas to induce or enhance chemical in situ processes. Emphasis will be on process mechanisms, preparation of materials, and potential applications. Papers presenting new, unpublished results are solicited on the following topics:

- Interaction mechanisms

Influence of photon ãnd energetic particle beams on surface chemistry, adsorption, desorption, migration, and nucleation

In situ diagnosis of interactions

- Photon and energetic particle beam induced or enhanced in situ processing Etching, deposition, doping, and surface modifications due to pyrolytic, photolytic, photo-electrochemical, and catalytic techniques

- Low-temperature growth of films, heterostructure, superlattices, etc.

- Applications

New beam concepts in lithography for wafer scale integration by direct writing or projection

Surface cleaning for UHV processing

In silu testing, customizing, and trimming of devices

Fabrication of new electronic and nonelectronic high tech materials

Symposium Chairs:

E.F. Krimmel

Siemens A.G

Otto-Hahn Ringo

D-8000 Munich 83

Federal Republic of Germany

(89) 636.44403

V.T. Nguyen

CNET GRENOBLE

BP. 98

F-38243 Meylan

France

76.51 .40 .6

\section{SHORT COURSE}

\section{Transmission Electron Microscopy of Materials June 8-12, 1987 \\ Massachusetts Institute of Technology Cambridge, MA}

This short course will prosent a comprehensive view of modern transmission and scanning transmission electron microscopy. Both lectures and laboratory exercises will provide the background and training necessary to bring the beginning microscopist to state-ofthe-art practice. The 15 hours of laboratory work will treat manipulation of the instruments, information to be gained from diffraction patterns, bright-field and dark-field microscopy, high resolution TEM, and microanalysis. Examples will be drawn from the areas of metals, ceramics, semiconductors, and polymers.

This short course will interest ind ustrial personnel engaged in microstructural analysis for research, development, or quality control purposes. In addition, the course may interest university researchers. students. staff or faculty who wish to be aware of current developments in electron microscopy. It is an appropriate in-depth sequel to the MRS introductory short course on TEM and can additionally serve as an effective introduction to an advanced course on analytical electron microscopy.

Application forms are available from: The Director. Summer Session. MIT, Room E19-356, Cambridge, MA 02139, 16171 253-2101; or from Prof. Linn HobDs, MIT, Room 13-4062, (617) 253-6835; or Prof. John Vander Sande, MIT, Room 13-5025, (617) 253-6933

\section{Simultaneous X-Ray Diffraction is at Booth 105.}

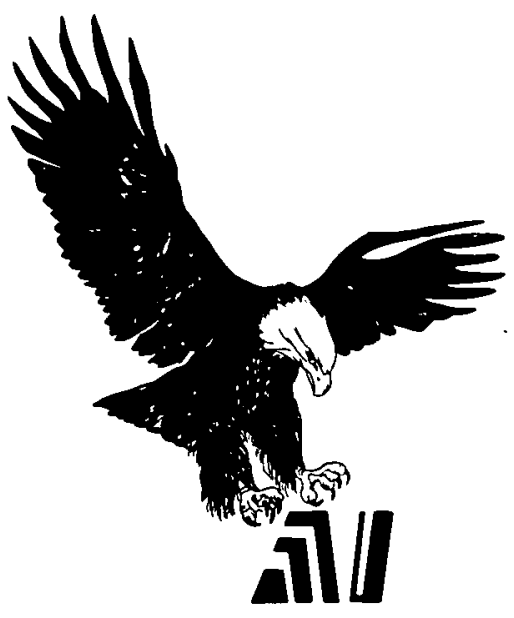

American Instruments, Inc. 185 Port Reading Ave., Port Reading, N.J. 07064 (201) 636-5770 Telex No. 5101006499 AIl NJ VISIT OUR BOOTH FOR FULL DETAILS. 


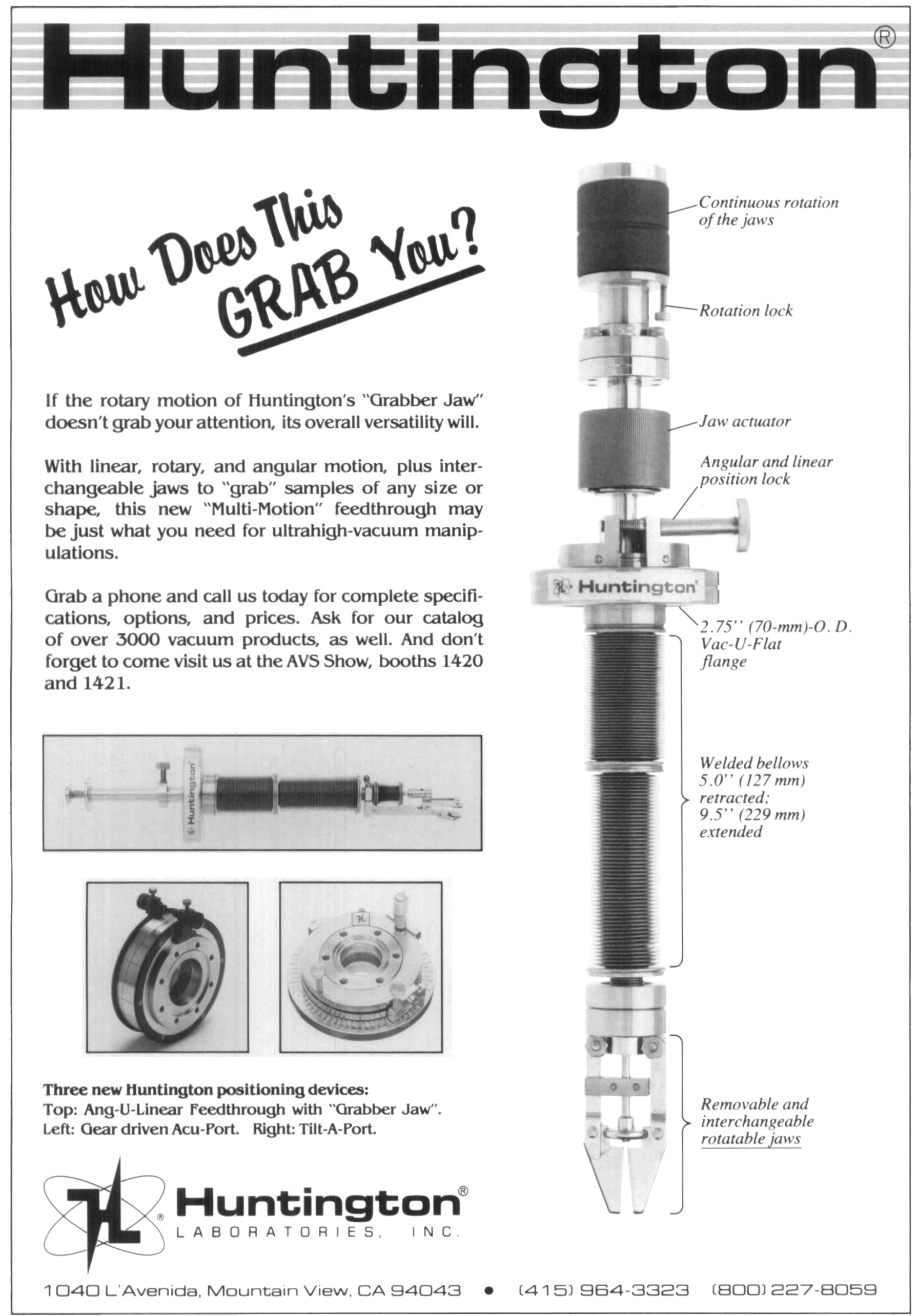

